\title{
SOME MODELLING ISSUES IN THE THEORY OF FRAGMENTATION-COAGULATION SYSTEMS*
}

\author{
JEAN-FRANÇOIS COLLET
}

\begin{abstract}
This paper is meant as an introduction to some of the most classical models in the theory of fragmentation-coagulation. The main models presented are the Becker-Döring, fragmentationcoagulation (discrete or continuous) and Lifshitz-Slyozov ones. Rather than focusing on mathematical technicalities, we have chosen to insist on the physical ideas behind their derivation, in order to present them in a unified framework. The unifying physical principle in this context is the mass action principle, which we expose in detail, our philosophy being that these models may be thought of as technical variations on this theme. We then present some qualitative properties of the models, which include saturation, criticality, and dissipation. The second part of the paper collects some mathematical tools which are of recurrent use in this context, namely the use of moments, of the Laplace transform, and of Lyapunov functions.
\end{abstract}

\section{Introduction: the physical picture}

In a very informal way, the systems under consideration here may be described as a population of particles which are free to move about in space and may merge after collisions, or spontaneously breakup. These two processes, respectively called coagulation and fragmentation in what follows, tend to modify the size and shape of the particles.

A vast amount of literature has been devoted to the study of the morphogenetic aspects of these systems, i.e. to the appearance and evolution of complex shapes that these dynamics may lead to (such as for instance dendritic growth induced by diffusion-limited aggregation, etc.).

We will however restrict the discussion here to situations where shape is not considered as a dynamical variable, so that the size profile of the system will be the fundamental quantity of interest. Our approach is therefore population-dynamical in spirit, and focuses on the time evolution of sizes.

Depending on the particular situation to be modelled the number of particles may be assumed to be finite or infinite, and their size may be treated as a discrete or continuous variable. Although this may lead to a variety of mathematical models, the physical nature of the corresponding systems is not fundamentally different.

Once these choices for the description of the number of particles and treatment of the sizes have been made, the derivation of a mathematical evolution model naturally proceeds in two steps:

1. Write a balance equation, which will involve some collision frequencies (or reaction rates in chemical terminology) as given coefficients;

2. Do some physics to obtain from physical assumptions on the dynamics of the interaction, an explicit expression for the coefficients.

This in principle leads to a closed model for the evolution of the size profile; interesting (and physically relevant) mathematical issues are then well-posedness, and qualitative properties of the corresponding Cauchy problem. Step 1 amounts to the expression of mass conservation, and basically consists of straightforward accounting with very little physics in it; Step 2 on the other hand requires a precise physical description of the agglomeration (or fragmentation) mechanism. In this connection it is maybe worthwhile pointing out that agglomeration mechanisms are significantly better doc-

*INRIA Projet OMEGA, 2004 route des Lucioles, BP 93, 06902 Sophia Antipolis, France (jfcollet@math.unice.fr). 
umented in the physical literature than their fragmentation counterpart. For the more mathematically inclined, Step 2 may be replaced by some assumption on the (abstract) coefficients, typically some form of bound, to ensure that the evolution is well-defined and has a certain number of properties.

\section{Part I}

\section{Some Modelling}

\section{Collision frequencies and reaction rates}

1.1. General mass action kinetics. The basic principle to be used here is the so-called mass action principle, which roughly says that the advancement rate of a chemical reaction is proportional to the concentrations of the involved reactants. To be more precise, let us consider a formal chemical reaction written as

$$
\sum_{i=1}^{n} \nu_{i} X_{i} \longrightarrow \sum_{i=1}^{n} \nu_{i}^{\prime} X_{i}
$$

Here the $X_{i}$ 's are arbitrary chemical species. For notational convenience all $X_{i}$ 's have been placed on both sides of the reaction, setting some of the coefficients to zero whenever necessary. This is harmless since the only quantities which will be involved in specifying the reaction velocities are not the stoichiometric coefficients $\nu_{i}$ but their differences $\alpha_{i}:=\nu_{i}^{\prime}-\nu_{i}$. Let us denote by $c_{i}$ the concentration of species number $i$; since we are only considering space-homogeneous situations (or equivalently, quantities at a fixed point in space), the reader unfamiliar with chemical language may think of this just as a number of molecules. The mass action principle then provides a system of ordinary differential equations for the concentrations:

$$
\frac{d c_{i}}{d t}=\alpha_{i} k \Pi_{j=1}^{n} c_{j}^{\nu_{j}}
$$

Here $k$ denotes the so-called rate constant, and again may be thought of just as a time frequency of reactive collisions. More precisely if $c_{i}$ denoted the (integer) number of molecules of species number $i$ in a space homogeneous system, $k$ would exactly represent the number of encounters of $\nu_{1}$ molecules of species $1, \nu_{2}$ molecules of species 2, and $\cdots$ and $\nu_{n}$ molecules of species $\mathrm{n}$ per unit time, to produce $\nu_{1}^{\prime}$ molecules of species $1, \nu_{2}^{\prime}$ molecules of species 2 , and $\cdots$ and $\nu_{n}^{\prime}$ molecules of species $n$.

Of course in the case of several simultaneous reactions, the time derivative $\frac{d c_{i}}{d t}$ is obtained by adding the contributions of all reactions, each of which is of the previous form. An important point to be stressed here is that in the time derivative of $c_{i}$, the only $i$-dependent term involved is the net gain $\alpha_{i}$.

1.2. The precise accounting for fragmentation-coagulation equations.

Let us apply mass-action kinetics as described in the previous paragraph, to an infinite system of discrete particles undergoing fragmentation and coagulation at the same time. This means that particles are defined by their size $i$, an integer which represents the number of elementary blocks (or "monomers" in the sequel) they are made of. These particles (or grains, or clusters in what follows) are free to merge or to 
break, which may be schematically represented by the doubly infinite set of chemical reactions:

$$
(i)+(j) \leftrightarrow(i+j), \quad i, j=1,2,3, \cdots
$$

If we focus our attention on one fixed size $i$, it is easy to see that this size is involved in two sets of reactions: on one hand, the ones written above with $i$ fixed and variable $j$, and on the other hand, the following:

$$
(i-k)+(k) \leftrightarrow(i)
$$

where $k$ ranges from 1 to $i-1$ (not to be taken in consideration if $i=1$ ). The accounting here is slightly complicated by the fact that in this second set of reactions the symetry obtaining upon exchanging $k$ and $i-k$ has to be taken into account. In other words the term corresponding to these reactions has to be divided by two... except in the case where $i$ is even, and $k=\frac{i}{2}$. Considering only coagulation for simplicity, we obtain the following expression for odd $i$ :

$$
\frac{d c_{i}}{d t}=\frac{1}{2} \sum_{j=1}^{i-1} k_{j, i-j} c_{j} c_{i-j}-\sum_{j=1, j \neq i}^{\infty} k_{i, j} c_{i} c_{j}-2 k_{i, i} c_{i}^{2}
$$

and for even $i$ :

$$
\frac{d c_{i}}{d t}=\frac{1}{2} \sum_{j=1, j \neq \frac{i}{2}}^{i-1} k_{j, i-j} c_{j} c_{i-j}+k_{\frac{i}{2}, \frac{i}{2}} c_{\frac{i}{2}}^{2}-\sum_{j=1, j \neq i}^{\infty} k_{i, j} c_{i} c_{j}-2 k_{i, i} c_{i}^{2}
$$

where $k_{i, j}$ is the collision frequency between particles of sizes $i$ and $j$. This somewhat unpleasant system may be recast in a simpler form. Indeed if one sets $k_{i, j}^{\prime}=k_{i, j}$ for $i \neq j$ and $k_{i, i}^{\prime}=2 k_{i, i}$, then (dropping of course the primes immediately) it assumes for any $i$ the usual form:

$$
\frac{d c_{i}}{d t}=\frac{1}{2} \sum_{j=1}^{i-1} k_{j, i-j} c_{j} c_{i-j}-\sum_{j=1}^{\infty} k_{i, j} c_{i} c_{j}
$$

A large number of articles devoted to this topic take this system as a starting point, but overlook the fact that $k_{i, i}$ is not equal to the frequency of $i$ - $i$ collisions, but its double. If this is of no importance for mathematical results which rely on boundedness assumptions for the coefficients, on the other hand it means that some results on explicit solutions are in error. It is of course very easy to include fragmentation, which yields the system:

$$
\frac{d c_{i}}{d t}=\frac{1}{2} \sum_{j=1}^{i-1}\left(k_{j, i-j} c_{j} c_{i-j}-q_{j, i-j} c_{i}\right)-\sum_{j=1}^{\infty}\left(k_{i, j} c_{i} c_{j}-q_{i, j} c_{i+j}\right) .
$$

In consistency with our notations for agglomeration coefficients, $q_{i, j}$ denotes the time frequency of fragmentations of $i+j$-particles into $i-$ and $j-$ particles for $i \neq j$, and twice that frequency for $i=j$. 
1.3. Some modelling for the collision frequencies. The relations derived so far are just balance equations, and we now give some indication on how the rate coefficients may be obtained from physical assumptions.

Coagulation seems to be in the present state better understood than fragmentation, so that we will restrict ourselves here to that case. For the case of fragmentation we refer the reader to the contribution of C. Baranger in this series. As was emphasized in the introduction, the expression for the collision frequency has to be derived from the study of the motion of the particles in physical space, and a description of the interaction mechanism. For simplicity we leave aside the physical chemistry of the interaction involved when particles meet, and concentrate on kinematics. This means that the collision frequency will be derived by computing the number of encounters per unit time, and assuming all collisions to be effective, that is to lead to agglomeration. These theoretical derivations typically proceed in two steps: first, consider a fixed $i$ - particle and compute the number of $j$-particles which hit it per unit time; then, take into account the fact that the $i$-particle itself is in motion. To give an indication of the sort of kernels that may arise, we will present three academic kinematic situations: flow-driven motion, Brownian motion, and sedimentation.

1.3.1. Flow-driven motion. If particles are advected by a fluid flow, collisions will result from velocity differences (an extreme case would be advection by some uniform constant velocity, in which case particles would never collide since they would all follow streamlines which do not intersect). This clearly shows that the driving mechanism here is velocity gradients. Therefore the simplest situation one can consider is that of a laminar shear flow, which we now consider as a case study (the following derivation is nothing but a slightly edited version of the original one given by Smoluchowski in [30]). A laminar shear flow is a flow which takes place along a fixed direction $x$, with a velocity $u$ depending linearly on some coordinate $z$ perpendicular to that direction: $u=(G z, 0,0)$, where $G$, the so-called shear rate, is a given positive constant. Consider a fixed spherical particle of radius $R_{i}$ located at the origin. A moving spherical particle of radius $R_{j}$ will stick to the fixed one as soon as the centers come to a distance $R_{i j}:=R_{i}+R_{j}$. In other words, the problem comes down to the computation of the number of point masses which hit the sphere $S_{i j}$ of radius $R_{i j}$ per unit time. This flux is easily computed by using spherical coordinates:

$$
z=r \cos \theta, x=r \cos \phi \sin \theta, y=r \sin \phi \sin \theta,-\frac{\pi}{2} \leq \theta \leq \frac{\pi}{2}, 0 \leq \phi \leq 2 \pi .
$$

Due to the symetry of the picture the incoming flux $J_{i j}$ on the sphere $S_{i j}$ is the flux on the area

$$
\{z \geq 0, x \leq 0\} \cup\{z \leq 0, x \geq 0\},
$$

or equivalently, twice the flux on the first part. We readily obtain

$$
\begin{aligned}
J_{i j} & =2 \int_{\theta=0}^{\frac{\pi}{2}} \int_{\phi=-\frac{\pi}{2}}^{\frac{\pi}{2}} u_{x} n_{x} d s \\
& =2 \int_{\theta=0}^{\frac{\pi}{2}} \int_{\phi=-\frac{\pi}{2}}^{\frac{\pi}{2}}\left(G R_{i j} \cos \theta\right)(\cos \phi \sin \theta)\left(R_{i j}^{2} \cos \theta\right) d \theta d \phi \\
& =\frac{4}{3} G R_{i j}^{3},
\end{aligned}
$$


hence the expression for the rate constant for this regime:

$$
k_{i j}=\frac{4}{3} G\left(R_{i}+R_{j}\right)^{3} .
$$

1.3.2. Brownian motion. We now consider the case of particles in Brownian motion. Here each $i$ - particle should be thought of as moving along a Brownian path with a diffusion coefficient given by the Stokes-Einstein formula:

$$
D_{i}=\frac{k T}{6 \pi R_{i} \mu},
$$

where $T$ is the absolute temperature, $k$ is Boltzmann's constant, and $\mu$ is the viscosity of the fluid. Again considering a fixed $i$-particle located at the origin, the problem is reduced to the computation of the flux of $j$ - particles through the sphere $S_{i j}$. The concentration $c_{j}$ of $j$ - particles in the medium surrounding the fixed $i$-particle evolves according to the diffusion equation:

$$
\frac{\partial c_{j}}{\partial t}=D_{j} \Delta c_{j}
$$

with appropriate boundary conditions at $r=R_{i j}$ and $r=\infty$ (we refer to [30] for details). This diffusion problem can then be solved in the steady state approximation to obtain that the number of $j$ - particles contacting the fixed one per unit time in a unit volume is given by:

$$
4 \pi\left(R_{i}+R_{j}\right) D_{j} c_{j}
$$

This now has to be corrected by taking into account the fact that the $i-$ particle itself is not fixed, but moves along Brownian paths with diffusion coefficient $D_{i}$. Assuming that particles do not interact during their free flights, the motions will be independent, so that the average relative displacement may be evaluated as follows:

$$
\overline{\left(\Delta x_{i}-\Delta x_{j}\right)^{2}}=\overline{\left(\Delta x_{i}\right)^{2}}+\overline{\left(\Delta x_{j}\right)^{2}}=\left(D_{i}+D_{j}\right) \Delta t
$$

This justifies the usual practice of replacing $D_{i}$ in (1.4) by the so-called "mutual diffusion coefficient" $D_{i}+D_{j}$. Using Einstein's formula we obtain the following expression for the Brownian coagulation rate:

$$
k_{i j}=\frac{2 k T}{3 \mu} \frac{\left(R_{i}+R_{j}\right)^{2}}{R_{i} R_{j}} .
$$

Let us note that this has the important consequence that in the case of a monodisperse solution (i.e. when all sizes are equal) we obtain the constant value

$$
k=k_{B}:=\frac{8 k T}{3 \mu}
$$

whatever the common size is. This justifies the classical approximation which consists in neglecting the dependence on size; however as was pointed out in section 2, the corresponding explicit solution usually found in the literature is in error, since in equation (1.3) the coefficient $k_{i, i}$ should be taken to be $2 k_{B}$, and all others set to $k_{B}$. 
1.3.3. Sedimentation. Sedimentation is the simplest possible situation in which particles velocities differ from that of the fluid. The picture here is a fluid at rest, in which particles fall under the action of gravity. Sticking in this context will occur because due to friction, particles with different radii fall with different velocities (as given by Stoke's formula, see [16] for details). The resulting coefficient is given by

$$
k_{i j}=\frac{2 \pi g}{9 \mu}\left(\rho_{s}-\rho\right)\left(R_{i}-R_{j}\right)\left(R_{i}+R_{j}\right)^{3},
$$

where $g$ is the gravity acceleration, $\mu$ is the dynamic viscosity of the fluid, $\rho_{s}$ is the density of the particles, and $\rho$ is that of the pure fluid (the derivation may be found in $[16])$.

\section{A few classical models}

System (1.3) may be thought of as the prototype for most models in fragmentationcoagulation. It is then very easy to design variations on this theme, by taking size as a continuous variable, placing restrictions upon the interaction coefficients, adding spatial dependence via transport or diffusion, adding more variables besides size such as energy or magnetization... The list of possible variations being endless (as well as the list of potential papers to be written on the corresponding Cauchy problems) we will now just present a few of the most classical models found in the physical literature.

Let us begin with the continuous size-version of (1.3). Denote by $x$ some measure of the size of the particles (such as their volume, or radius if they are assumed to be spherical) and by $f(t, x)$ the size-density of the system. The continuous analog of (1.3) is now readily obtained by replacing the discrete sums with integrals:

$$
\begin{aligned}
\frac{\partial}{\partial t} f(t, x) & =\frac{1}{2} \int_{0}^{x}[k(x-y, y) f(x-y, t) f(y, t)-q(x-y, y) f(x, t)] d y \\
& -\int_{0}^{\infty}[k(x, y) f(x, t) f(y, t)-q(x, y) f(x+y, t)] d y .
\end{aligned}
$$

Here the so-called "coagulation and fragmentation kernels" $k(x, y)$ and $q(x, y)$ are the continuous analogs of the coefficients $k_{i, j}$ and $q_{i, j}$, and again have the physical meaning of time frequencies.

An interesting particular case of (1.3), the so-called Becker-Döring model, is obtained by assuming that only monomers can be captured or shed at a time. Mathematically this means taking:

$$
k_{i, j}=q_{i, j}=0 \quad \text { for } \quad \min (i, j)>1 .
$$

Changing slightly the notations we set

$$
a_{i}:=k_{i, i+1}, \quad b_{i}:=q_{i, i-1} .
$$

The system now becomes

$$
\begin{gathered}
\frac{d c_{i}}{d t}=J_{i-1}-J_{i} \\
\frac{d c_{1}}{d t}=-2 J_{1}-\sum_{i=2}^{\infty} J_{i} .
\end{gathered}
$$


Here we have denoted by $J_{i}$ the velocity of the reaction:

$$
(i)+(1) \leftrightarrow(i+1), \quad i \geq 1,
$$

that is to say:

$$
J_{i}=a_{i} c_{1} c_{i}-b_{i+1} c_{i+1} \quad \text { for } \quad i \geq 1 .
$$

This is the Becker-Döring system, introduced in [6]. The interested reader will find a very complete mathematical theory of this system in [5]. Obviously if the total mass is to be preserved, the following relation should hold true:

$$
\frac{d}{d t} \sum_{i=1}^{\infty} i c_{i}(t)=0 .
$$

This can be formally derived by multiplying (2.3) by $i$ and then summing over $i$, so that (2.4) may be replaced by (2.5). It is shown in [5] that the physically reasonable solutions to systems (2.3)-(2.4) on one hand and (2.3)-(2.5) on the other hand, are unique, and are the same, so that from now on what we refer to as the Becker-Döring system is the following:

$$
\begin{array}{r}
\frac{d c_{i}}{d t}=\left[a_{i-1} c_{1} c_{i-1}-b_{i} c_{i}\right]-\left[a_{i} c_{1} c_{i}-b_{i+1} c_{i+1}\right] \quad i \geq 2, \\
\sum_{i=1}^{\infty} i c_{i}(t)=\rho .
\end{array}
$$

From a formal mathematical point of view, it may look from (2.2) as though (2.6)(2.7) is just a particular case of (1.3). From the physical point of view however, let us emphasize that this situation should be thought of as a different regime from the full fragmentation-coagulation regime, or more precisely as an earlier stage. Think of an initially monodisperse population of grains (call them monomers), which for some reason are unable to fragment. In the early stage of the evolution, these monomers will interact to build larger grains, but will still constitute the bulk of the population, in such a way that the reactions

$$
(i)+(1) \leftrightarrow(i+1)
$$

will dominate. At a later stage however there will be enough large (here large means $i>1$ ) grains so that they may interact between themselves, and possibly fragment, so that the full system (1.3) has to be taken into account. The earlier stage is the socalled nucleation stage, and the later one is the so-called coalescence, or fragmentationcoalescence stage.

As before, size may be treated as a continuous variable in the nucleation stage. Let us denote by $x$ the volume variable, and by $f(t, x)$ the density with respect to $x$. The continuity equation reads

$$
\frac{\partial f}{\partial t}+\frac{\partial J}{\partial x}=0 \quad x>0, t>0,
$$

where $J(x, t)$ denotes the flux in $x$-space. This equation expresses the mass balance for large grains; it has to be coupled to the relation expressing total mass conservation:

$$
c(t)+\int_{0}^{\infty} x f(t, x) d x=\text { const },
$$


where $c(t)$ denotes the concentration of monomers in the bath. In such a mean field description, the flux of clusters in size space may or may not include a diffusive part, depending on whether or not one decides to take fluctuations into account. If fluctuations are not taken into account one deals with the pure transport regime, in which the flux is given by $J(x, t)=f(x, t) G(x, t)$, where $G$ denotes the growth velocity of a grain of volume $x$. In complete analogy with fluid mechanics (replacing the position variable by the size variable), if one thinks of $x$ and $t$ as Lagrangian coordinates, $G$ represents the Lagrangian velocity. In physical language this means that a fixed macroscopical grain of size $x$ grows according to $\frac{d x}{d t}=G(x, t)$.

The precise expression for $G$ has to be derived from physical assumptions on the kinetics of the interaction between large grains and monomers. Assuming that large grains are a minority phase, this depends on how monomers move about, and how they attach to (or are liberated from) large grains. Perhaps the most studied case is that of the diffusion-limited regime, in which monomers diffuse around crystals, the equilibrium concentration being given by the Gibbs-Thompson formula. This yields the celebrated Lifshitz-Slyozov system (first derived in the seminal paper [26]; see also [33]):

$$
\begin{aligned}
\left.\frac{\partial f}{\partial t}+\frac{\partial}{\partial x}\left(\left(c(t) x^{\frac{1}{3}}-1\right) f(x, t)\right)\right) & =0 \\
c(t)+\int_{0}^{\infty} x f(t, x) d x & =\rho .
\end{aligned}
$$

Here again $x$ is the volume variable, $c(t)$ is the concentration of monomers in the bath at time $t$, and as before $f$ is the density of grains with respect to the $x$ variable.

\section{Some qualitative features of the dynamics}

3.1. Lifshitz-slyozov: critical radius and ostwald ripening. From relation (2.9) above one sees that at any fixed time, the growth velocity of grains vanishes and changes sign at the so-called critical volume $v_{c}$ defined by:

$$
v_{c}(t)^{\frac{1}{3}}=\frac{1}{c(t)} .
$$

The interesting point here is that supercritical grains (i.e. grains whose volume is larger than $v_{c}$ ) grow, whereas subcritical ones shrink. One might then think that the dynamics are trivial in the sense that the profile continuously gets shifted towards large volumes, but here mass conservation provides a natural feedback: as large grains grow the solution gets depleted in monomers, thus $c$ decreases, and therefore the critical volume increases, so that supercritical grains become in fact "less supercritical" as time passes (the precise inequalities on the characteristic velocities which translate this idea may be found in [28]). This is the essence of the so-called phenomenon of "Ostwald Ripening". Since we have a system maintained out of equilibrium in the presence of a nonlinear feedback, it is natural to expect that some interesting patterns will develop; the paper [26] presents qualitative arguments supporting the idea that some nontrivial intermediate asymptotics (i.e. self-similar behaviour) will develop, and these arguments have since then been refined in [24].

3.2. Becker-Döring: saturation and "weak" phase transition. Let us now turn to the Becker-Döring system, and in particular to its steady-state solutions. From the physical principle of micro-reversibility we are interested in solutions for 
which all reactions are turned off. Denoting by $\left(m_{i}\right)_{i \in \mathbb{N}}$ such a solution, we obtain

$$
m_{i}=Q_{i}\left(m_{1}\right)^{i}, \text { where } \quad Q_{1}=1, \quad Q_{i}=\prod_{k=1}^{i-1}\left(\frac{a_{k}}{b_{k-1}}\right) \quad i \geq 2 .
$$

Thus we obtain a one-parameter family of equilibria, parametrised by the value of the monomer concentration $m_{1}$. We can then compute the total mass associated to $m$ :

$$
\rho=\sum_{i=1}^{\infty} i m_{i}=\sum_{i=1}^{\infty} i Q_{i}\left(m_{1}\right)^{i} .
$$

All coefficients being positive, this is a strictly increasing function of $m_{1}$, so that the family of equilibria may be parametrised by the total mass $\rho\left(m_{1}\right)$. The interesting fact is that for such an equilibrium to exist, the monomer concentration has to be less than the radius of convergence of the power series (3.2), which we will denote by $c_{s}$ :

$$
m_{1} \leq c_{s}:=\left(\limsup _{i}\left(Q_{i}\right)^{1 / i}\right)^{-1}
$$

If the kinetic coefficients are such that this convergence radius is finite, then the corresponding mass

$$
\rho_{s}:=\sum_{i=1}^{\infty} i Q_{i}\left(c_{s}\right)^{i}
$$

corresponds to the saturated vapor density: that means, the maximum mass which the system can withstand without trying to manufacture a new phase (see the section on asymptotic behaviour, lower, for more details on this).

3.3. Change of scale and "Hydrodynamic" limits. As was explained above the Becker-Döring and Lifshitz-Slyozov regimes are physically different, and correspond respectively to the nucleation and coalescence stage - a point completely misunderstood by many mathematicians working in this area. In the nucleation stage fluctuations still play an important role, and there is no notion of critical size. In the (later) coalescence stage there is a well-defined critical size, and grains move in size-space in a deterministic fashion, depending on whether they are supercritical or subcritical. In a more probabilistic language, system $(2.6)(2.7)$ has the flavor of the Kolmogoroff equation of some Markov process which acts on sizes, whereas (2.9)(2.10) describes a pure transport regime. If one thinks of the similarity with the various possible levels of description of fluids (i.e. many-particle, kinetic and fluid), it is on the other hand natural to ask whether $(2.9)(2.10)$ may be obtained from $(2.6)(2.7)$ via some change-of-scale argument (The mathematicians alluded to above would however just think of $(2.6)(2.7)$ as a finite difference approximation to $(2.9)(2.10))$.

Various approaches to this issue may be found in [9],[35] and [14]. As the reader familiar with hydrodynamic limits will expect, a scaling argument for the BeckerDöring yields the transport term of the Lifshitz-Slyozov equation as a leading term, and an $\epsilon$ - order viscosity correction. More precisely, if one introduces a rescaling parameter $\epsilon$ in the Becker-Döring system in order to view the right hand side of (2.7) as a difference quotient (therefore a flux divergence in the limit of vanishing $\epsilon$ ), the equivalent equation may be shown to be the following:

$$
\frac{\partial}{\partial t} f+\frac{\partial}{\partial x}\left[(a(x) c(t)-b(x)) f-\epsilon \frac{\partial}{\partial x}\left(\frac{a(x) c(t)+b(x)}{2} f(t, x)\right)\right]=0 .
$$


This model was introduced with formal arguments in [12]; a rigorous derivation of the equation, as well as appropriate boundary conditions, may be found in [14]. This result is in a sense comparable to a diffusive hydrodynamic limit; to pursue the analogy with fluid mechanics, let us remark that the corresponding drift-diffusion problem has not been yet investigated. More precisely, it would be interesting to give a rigorous derivation of either (2.6)-(2.7) or (3.5) from (1.3), much in the way one can go from a kinetic equation to a drift-diffusion equation (see [25] for details). Alternately one could start from the continuous version (2.1), and rescale the interaction kernel.

\section{Moments}

Considering the discrete-size case, for instance $(2.6)(2.7)$ or (1.3), it is quite easy to understand why moments of the solution, i.e. quantities of the form

$$
m_{\alpha}(t):=\sum_{i=1}^{\infty} i^{\alpha} c_{i}(t)
$$

should come very handy in the mathematical analysis. From the mass conservation relation (2.7) it appears that a natural space to be working in is the Banach space $w^{1,1}$ (our notation is chosen to emphasize the analogy with Sobolev spaces) of sequences $\left(c_{i}\right)_{i \in \mathbb{N}}$ such that the quantity $\sum_{i=1}^{\infty} i\left|c_{i}\right|$ (which turns out to provide a norm) is finite. Most results on existence or asymptotic behaviour rely on some compactness property, which in the space $w^{1,1}$ is obtained by bounding from above some moment of the form (4.1) with $\alpha>1$. More generally, we will be interested in the time evolution of some quantities associated to the size profile, which in the discrete size case for instance take the form:

$$
\Phi(c):=\sum_{i=1}^{\infty} \phi_{i} c_{i}
$$

For instance upon taking $\phi_{i}=i$, the quantity $\Phi$ represents the total mass of the system, or with $\phi_{i}=i^{\frac{2}{3}}$ it represents the total area of the grains, and finally just like in kinetic theory, the choice $\phi_{i}=\log c_{i}$ gives the entropy of the system. To illustrate this, let us consider system (1.3): we immediately get

$$
\frac{d \Phi}{d t}=\frac{1}{2} \sum_{k=1}^{\infty} \phi_{k} \sum_{j=1}^{k-1}\left(k_{k-j, j} c_{k-j} c_{j}-q_{k-j, j} c_{k}\right)-\sum_{k=1}^{\infty} \phi_{k} \sum_{j=1}^{\infty}\left(k_{k, j} c_{k} c_{j}-q_{k, j} c_{k+j}\right) .
$$

Fubinizing the first sum and symetrizing the second one we obtain the relation:

$$
\frac{d \Phi}{d t}=\frac{1}{2} \sum_{k=1}^{\infty} \sum_{j=1}^{\infty}\left(\phi_{k+j}-\phi_{k}-\phi_{j}\right)\left(k_{k, j} c_{k} c_{j}-q_{k, j} c_{k+j}\right) .
$$

The physical meaning of this relation is obvious, since the quantity $\phi_{k+j}-\phi_{k}-\phi_{j}$ represents the net increase of the quantity $\phi$ during one elementary step $(i)+(j) \rightarrow(i+$ $j)$, and the corresponding factor $\left(k_{k, j} c_{k} c_{j}-q_{k, j} c_{k+j}\right)$ is the number of such elementary steps per unit time. In stochastic parlance, the right hand side of (4.2) defines the action on $\phi$ of the infinitesimal generator of a Markov semigroup associated to the transitions (1.2). Of course as a byproduct we obtain (formally) mass conservation:

$$
\frac{d}{d t} \sum_{i=1}^{\infty} i c_{i}=0
$$


The continuous analog is straightforward: defining $\Phi(t)$ by

$$
\Phi(t):=\int_{0}^{\infty} \phi(x) f(t, x) d x
$$

any solution to (2.1) will satisfy

$\frac{d \Phi}{d t}=\frac{1}{2} \int_{0}^{\infty} \int_{0}^{\infty}[\phi(x+y)-\phi(x)-\phi(y)][k(x, y) f(y, t) f(x, t)-q(x, y) f(x+y, t)] d x d y$.

Besides providing the time evolution of quantities of this type, note that this formulation may be readily used to define the notion of solution to the agglomeration systems even in a measure-valued context. More precisely let us consider the pureagglomeration system obtained by taking all fragmentation coefficients to be zero in (2.1):

$$
\frac{\partial}{\partial t} f(t, x)=\frac{1}{2} \int_{0}^{x} k(x-y, y) f(x-y, t) f(y, t) d y-\int_{0}^{\infty} k(x, y) f(x, t) f(y, t) d y .
$$

A time-dependent measure $\mu$ will be said to be a solution to (4.4) if for all $t>0$, and any $\phi$ in a suitable class of test functions, the following relation:

$$
\frac{d}{d t} \int_{0}^{\infty} \phi(x) \mu(d x, t)=\frac{1}{2} \int_{0}^{\infty} \int_{0}^{\infty}[\phi(x+y)-\phi(x)-\phi(y)] k(x, y) \mu(d y, t) \mu(d x, t) .
$$

holds true (we refer to the lecture by J.Norris in this series for a study of this equation).

In more physical (and pompous) terminology, this is a "principle of virtual work" for chemical kinetics.

\section{Some exact solutions: the laplace transform trick}

The Laplace transform may be used to obtain explicit solutions for some particular kinetic coefficients, as well as to show convergence to some self-similar profile for large time. The general methodology here is to derive some integro-differential equation for the Laplace transform of the size profile (since agglomeration mathematically amounts to taking a convolution product, it should come as no surprise that the Laplace transform has a role to play here). In the case where the coefficients have a power dependence on size this equation turns out to be a partial differential equation which may sometimes be solved explicitly. The size profile may then be recovered by just taking an inverse Laplace transform. Besides providing an interesting calculus exercise, these computations lead to the very natural issue of stability of these profiles with respect to the coefficients. Let us now illustrate the method on the simplest possible example, the discrete agglomeration equation:

$$
\frac{\partial c_{i}}{\partial t}=\frac{1}{2} \sum_{j=1}^{i-1} k_{j, i-j} c_{j} c_{i-j}-c_{i} \sum_{j=1}^{\infty} k_{i, j} c_{j} .
$$

We introduce the associated "generating series", i.e. the discrete Laplace transform of the sequence $\left(c_{i}(t)\right)_{i}$ with respect to $i$ :

$$
G(x, t)=\sum_{k=1}^{\infty} c_{k}(t) \exp (k x)
$$


The partial derivatives of $G$ are given by:

$$
\frac{\partial^{p} G}{\partial x^{p}}(x, t)=\sum_{k=1}^{\infty} k^{p} c_{k}(t) \exp (k x) .
$$

The keypoint here is that the time derivative of $G$ may be expressed by setting $\phi_{k}:=\exp k x$ in $(4.2)$ :

$$
\frac{\partial G}{\partial t}(x, t)=\frac{1}{2} \sum_{k=1}^{\infty} \sum_{j=1}^{\infty}\left(e^{(k+j) x}-e^{k x}-e^{j x}\right) k_{k j} c_{j}(t) c_{k}(t) .
$$

In this relation, the right hand side turns out to be an exact derivative of $G$ in the case where $k_{i j}$ is a power of $i j$.

In cases where the equation for $G$ may be solved explicitly, the profile may then be obtained by an inverse Laplace transform:

$$
k^{p} c_{k}(t)=\frac{1}{2 \pi} \int_{-i \pi}^{i \pi} e^{-k x} \frac{\partial^{p} G}{\partial x^{p}}(x, t) d x .
$$

Let us begin by the simplest case $k_{i j}=1$ for all $i, j$; equation (5.2) becomes:

$$
\frac{\partial G}{\partial t}(x, t)=\frac{1}{2} G(x, t)^{2}-G(0, t) G(x, t) .
$$

This may be solved explicitly to yield:

$$
G(x, t)=\frac{G(x, 0)}{\left(1+\frac{\gamma_{0} t}{2}\right)\left(1-\frac{t}{2}\left(G(x, 0)-\gamma_{0}\right)\right)},
$$

where $\gamma_{0}:=G(0,0)$. Note that here the initial value $G(x, 0)$ is known:

$$
G(x, 0)=\sum_{k=1}^{\infty} c_{k}(0) \exp (k x)
$$

The concentrations $c_{k}$ may then be recovered by inversion formula, and their behaviour for large time may be then directly read-off from the explicit formula. This method works equally well for the case of additive and multiplicative coefficients, and we refer the reader to [18] for details.

In the continuous case, let us indicate a nice computational device which is very close to the usual Laplace transform. We consider (2.1) in the absence of fragmentation, i.e. we set $q(x, y)=0$ :

$$
\frac{\partial f}{\partial t}=\frac{1}{2} \int_{0}^{x} k(y, x-y) f(y, t) f(x-y, t) d y-f(x, t) \int_{0}^{\infty} k(x, y) f(y, t) d y .
$$

We now set

$$
N(p, t):=\int_{0}^{\infty} f(x, t) \phi_{p}(x) d x
$$

with the notation

$$
\phi_{p}(x):=\exp (-p x)-1
$$


This device may be found in [23], and was used later in [27] under the name "desingularized Laplace transform". The beauty of subtracting 1 to the integrand in the usual definition of the Laplace transform is that $\phi_{p}$ satisfies:

$$
\phi_{p}(x+y)-\phi_{p}(x)-\phi_{p}(y)=\phi_{p}(x) \phi_{p}(y) .
$$

Combining (4.3) and (5.5) we see that $N$ satisfies the following relation:

$$
\frac{\partial N}{\partial t}(p, t)=\frac{1}{2} \int_{0}^{\infty} \int_{0}^{\infty} f(x, t) f(y, t) \phi_{p}(x) \phi_{p}(y) k(x, y) d x d y .
$$

This relation turns out to be a Partial Differential Equation for $N$ in the case where $k$ has a simple homogeneity property. For instance if $k=1$ we obtain

$$
\frac{\partial N}{\partial t}=\frac{1}{2} N^{2}
$$

(compare to (5.4)). In the case where $k(x, y)=x y$, noting that

$$
\frac{\partial N}{\partial p}(p, t)=-\int_{0}^{\infty} x \exp (-p x) f(x, t) d x
$$

we obtain the equation

$$
\frac{\partial N}{\partial t}(p, t)=\frac{1}{2}\left(\frac{\partial N}{\partial p}(p, t)-\frac{\partial N}{\partial p}(0, t)\right)^{2},
$$

which may be integrated explicitly. In either (constant or additive) case, the integrated version of this equation involves the Laplace transform of the initial profile, which can only be computed for simple enough data (a large number of articles in the applied literature deal with a monodisperse initial state). Without delving too much into details, let us just say that this method provides explicit solutions for initial data which are simple enough (i.e. whose Laplace transform is known), and for kinetic coefficients which are simple enough (mostly, constant, additive, and multiplicative).

\section{Asymptotic behaviour via Lyapunov functions}

6.1. Motivation: intermediate versus final asymptotics. As regards asymptotic behaviour, two types of asymptotics may be studied here: the so-called "intermediate", and "final" one. This is a quite typical feature in the study of dissipative dynamical systems. To illustrate the point, let us consider the simplest example which exhibits this sort of behaviour, namely the heat equation in the whole space. For simplicity we look at a one-dimensional situation, although the behaviour is qualitatively dimension-independent:

$$
\frac{\partial u}{\partial t}=\frac{1}{2} \frac{\partial^{2} u}{\partial t^{2}}, \quad x \in \mathbb{R}, t>0 .
$$

It is very easy to show that for any fixed $x \in \mathbb{R}$ the solution goes to zero for large time:

$$
u(x, t) \rightarrow 0, \quad t \rightarrow \infty .
$$

This is the "final", or trivial, asymptotics. However a proper rescaling of the solution has a non trivial large-time behaviour. The precise result in one spatial dimension is the following:

$$
\sqrt{t} u(x \sqrt{t}, t) \rightarrow C \exp \left(-\frac{x^{2}}{2 t}\right), \quad t \rightarrow \infty
$$


where $C$ is a constant which is uniquely determined from the initial data (via mass conservation). Of course in such a simple case this behaviour may be directly read-off from the explicit representation of the solution in terms of the heat kernel. Such intermediate asymptotic results are however valid for more general equations, in which case they may be derived by using either relative entropy $[32,36]$ or renormalization-group methods [7]. To summarize, some rescaled version of the solution has an interesting behaviour for large time, in the sense that it converges to some unique point in a class of functions which depend on a small number of parameters. This number is equal to the number of quantities which are prescribed by the dynamics (here one, the total space integral), and the selected values for the parameters are uniquely determined from the conserved quantities. Let us now indicate the relevance (or lack thereof) of this scenario in the context of agglomeration systems.

6.2. Mass action kinetics as a dissipative mechanism and the invariance principle. The main tool here is Lyapunov functions, which roughly means, quantities which decrease along the flow (the precise definitions and results will be given below for the reader's convenience). Let us take a step back and consider the general chemical reaction (1.1), which will be supposed to take place both in the forward and backward directions. Denoting respectively by $k_{f}$ and $k_{b}$ the corresponding rates, the differential system for the chemical concentrations will be:

$$
\frac{d c_{i}}{d t}=\alpha_{i} k_{f} \Pi_{j=1}^{n} c_{j}^{\nu_{j}}-\alpha_{i} k_{b} \Pi_{j=1}^{n} c_{j}^{\nu_{j}^{\prime}}=\alpha_{i} k_{b} \Pi_{j=1}^{n} c_{j}^{\nu_{j}}\left(K-\Pi_{j=1}^{n} c_{j}^{\alpha_{j}}\right),
$$

where the equilibrium constant $K$ is defined by $K=\frac{k_{f}}{k_{b}}$. An array $\left(m_{i}\right)$ of equilibrium concentrations therefore has to satisfy the relation:

$$
\Pi_{j=1}^{n} m_{j}^{\alpha_{j}}=K .
$$

Assuming that such a point $m$ exists, the time-derivative of $c_{i}$ may now be rewritten:

$$
\frac{d c_{i}}{d t}=\alpha_{i} k_{b} \Pi_{j=1}^{n} c_{j}^{\nu_{j}}\left(\Pi_{j=1}^{n} m_{j}^{\alpha_{j}}-\Pi_{j=1}^{n} c_{j}^{\alpha_{j}}\right):=\alpha_{i} \frac{d \xi}{d t},
$$

where the last equality serves as a definition (within an additive constant) for the advancement of the reaction $\xi$. If we define the entropy (which should in fact be more appropriately be called free energy here) by

$$
H(t)=\sum_{i=1}^{n} c_{i}(t)\left(\log \frac{c_{i}}{m_{i}}-1\right)
$$

it is very easy to check that its time-derivative is given by

$$
\frac{d H}{d t}=k_{b}\left(\Pi_{j=1}^{n} c_{j}^{\nu_{j}}\right)\left(\log \Pi_{j=1}^{n} c_{j}^{\alpha_{j}}-\log \Pi_{j=1}^{n} m_{j}^{\alpha_{j}}\right)\left(\Pi_{j=1}^{n} m_{j}^{\alpha_{j}}-\Pi_{j=1}^{n} c_{j}^{\alpha_{j}}\right),
$$

and is therefore nonpositive. This dissipation property is a fundamental feature of chemical systems. It is in fact (as well as the very form of the entropy production term) in striking analogy with the famous $H$-theorem of Boltzmann, and may be used to infer the stability of the equilibrium $m$. The important point here is that not only does $H$ decrease along orbits, but its time derivative vanishes only at the 
equilibrium point. This strongly suggests that the orbit converges to this equilibrium point for large time, which can be rigorously proved by the use of the well-known invariance principle. Let us now recall the precise mathematical tools which may be used to carry out this program; the interested reader may consult [20], [3] and[4] for more details.

Recall that a dynamical system, or a flow on a complete metric space $X$ is a family of continuous maps $(S(t))_{t \geq 0}$ from $X$ into itself ( $t$ should be thought of as the time variable), such that for any point $x \in X$ the trajectory $t \mapsto S(t) x$ is continuous from $[0, \infty[$ into $X$, and which enjoy the usual semigroup property:

$$
S(0)=I d_{X}, \quad S(t+s)=S(t) S(s), \quad \forall s, t \geq 0 .
$$

A subset $K$ of $X$ is said to be invariant under the flow if for any point $x_{0} \in K$ there exists a curve $x: \mathbb{R} \rightarrow K$ such that $x(0)=x_{0}$, and

$$
S(t) x(s)=x(t+s) \quad \text { for all } \quad t \geq 0, s \in \mathbb{R} .
$$

This merely expresses the idea that the complete (i.e. for both positive and negative times) orbit $x(t)$ passing through $x_{0}$ at time zero is defined and lies entirely in $K$. Finally a Lyapunov function for the flow is a continuous real-valued function $H$ defined on $X$, such that for any $x \in X$ the quantity $\dot{H}(x)$ defined by

$$
\dot{H}(x):=\limsup _{t \rightarrow 0^{+}} \frac{H(S(t) x)-H(x)}{t}
$$

is nonpositive. Here the idea is to look at the time-derivative of $H$ along the orbit, and note that by taking the limsup we ensure that $V$ decreases along the orbit, without necessarily being differentiable. We may now state the simplest version of the invariance principle:

Theorem 6.1. Let $X$ be a complete metric space, and $(S(t))_{t \geq 0}$ a flow on $X$, endowed with a Lyapunov function $H$. Let $E$ be the set of points of $X$ where $\dot{H}$ vanishes, and $M$ be the largest invariant set contained in $E$. Let $x_{0}$ be a point in $X$, and assume that the positive orbit through $x_{0}$ lies in a compact set of $X$. Then the conclusion is that it approaches $M$ for large times:

$$
S(t) x_{0} \rightarrow M \quad \text { as } \quad t \rightarrow \infty .
$$

In many applications the flow has an isolated equilibrium point $x^{*}$, which also is an isolated zero of $\dot{H}$, and which is stable in the sense that any orbit started in a certain neighborhood $V$ of $x^{*}$ remains in it. Considering the flow restricted to $V$ and noting that $M=E=\left\{x_{0}\right\}$, the immediate conclusion is that all orbits that started in $V$ converge to $x^{*}$ for large times.

\subsection{Final asymptotics: the H-Theorem for various agglomeration sys-} tems. Having seen that mass action kinetics is a dissipative mechanism, it is natural to expect that the various systems presented above should have Lyapunov functions. This is indeed the case, and we now give some of them. Starting with the Becker-Döring system $(2.6)(2.7)$, assume that the total mass $\rho$ is less than the saturation mass $\rho_{s}$ defined by (3.4), so that a detailed balance equilibrium $m=\left(m_{i}\right)_{i \geq 1}$ defined by (3.1) exists (it is therefore unique). Setting

$$
H(c)=\sum_{i=1}^{\infty} c_{i}\left(\ln \frac{c_{i}}{m_{i}}-1\right)
$$


It is very easy to check that any solution to the Becker-Döring system satisfies

$$
\frac{d}{d t} H(c(t))=\sum_{i=1}^{\infty}\left(a_{i} c_{i} c_{1}-b_{i+1} c_{i+1}\right) \ln \frac{b_{i+1} c_{i+1}}{a_{i} c_{i} c_{1}} \leq 0 .
$$

This was used in [5] to infer that for large time:

$$
c_{i}(t) \rightarrow m_{i}
$$

This being only a qualitative conclusion, one may then ask about the rate of convergence. It is quite well-known now that for many dissipative systems, some logarithmic Sobolev inequality may be used to derive a Gronwall estimate for the entropy, which then in turns yields a convergence rate. This is typically the case when the equilibrium state is given by a Gaussian distribution, for which a logarithmic Sobolev inequality is valid. Of course the Becker-Döring equilibria do not fall into that category, but it is quite interesting to note that this scheme of proof was used successfully in [22], where the authors obtain a rate for the convergence to the final state, by using a weaker inequality.

The final state is somehow a trivial regime. However here an interesting thing happens in the case where one imposes supersaturated initial data. Assume that the saturation mass $\rho_{s}$ defined by (3.4) is finite, and take initial data which are supersaturated, that is with total mass $\rho$ larger than $\rho_{s}$. In this situation the dissipation is still present, but the total mass cannot be identified. More precisely if one knows that there is convergence to some detailed balance equilibrium:

$$
c_{i}(t) \rightarrow Q_{i} m_{1}^{i} \quad i=1, \cdots,
$$

then in order to identity the corresponding $m_{1}$ one feels like using the string of equalities

$$
\rho=\lim _{t \rightarrow \infty} \sum_{i=1}^{\infty} i c_{i}(t)=\sum_{i=1}^{\infty} i \lim _{t \rightarrow \infty} c_{i}(t)=\sum_{i=1}^{\infty} i Q_{i}\left(m_{1}\right)^{i} .
$$

The keypoint here is that being on the circle of convergence prevents us from inverting the pointwise limit and the infinite sum, hence the second of these equalities is false, so that the knowledge of $\rho$ is not sufficient to identify the selected value for $m_{1}$. What happens then is that the excess mass $\rho-\rho_{s}$ runs away to infinity, and is asymptotically lost; the precise convergence result may be found in [5], but suffice it here to say that for supercritical data, the usual folklore exposed above on the heat equation does not apply: knowledge of the conserved quantities is not sufficient to identify the limit.

Let us now turn to the general abstract fragmentation-coagulation system (1.3). Let us assume that a detailed balance equilibrium, i.e. an array $m$ of concentrations for which all reactions are turned off, exists. This means that $m$ satisfies the infinite set of relations:

$$
a_{i, j} m_{i} m_{j}=b_{i, j} m_{i+j}, \quad i, j=1, \cdots \infty
$$

(note that the mere assumption that such an $m$ exists places very severe restrictions on the kinetic coefficients). Defining the entropy $H$ by

$$
H(t)=\sum_{i=1}^{\infty} c_{i}(t) \log \frac{c_{i}}{m_{i}}
$$


and using (4.2) with $\phi_{i}=\log \frac{c_{i}}{m_{i}}$, we obtain:

$$
\frac{d H}{d t}=\frac{1}{2} \sum_{i=1}^{\infty} \sum_{j=1}^{\infty} k_{i, j} m_{i} m_{j}\left(\log \frac{c_{i+j}}{m_{i+j}}-\log \frac{c_{i} c_{j}}{m_{i} m_{j}}\right)\left(\frac{c_{i} c_{j}}{m_{i} m_{j}}-\frac{c_{i+j}}{m_{i+j}}\right) \leq 0 .
$$

Note that in fact, the Becker-Döring case is just a particular case of this one. In either case, the physical meaning of the quantity $H$ is that of free energy (recall the famous relation in classical thermodynamics relating the chemical potential to the logarithm of the concentration). Let us conclude this section with the Lifshitz-Slyozov system, for which the Lyapunov function has a very different meaning. Starting from (2.9)(2.10) we put

$$
H(t)=3 \int_{0}^{\infty} x^{\frac{2}{3}} f(t, x) d x+c(t)^{2} .
$$

It is then straightforward to check that

$$
\frac{d K}{d t}=-2 \int_{0}^{\infty} x^{\frac{-1}{3}}\left(1-c(t) x^{\frac{1}{3}}\right)^{2} f(t, x) d x \leq 0 .
$$

This fact was used in [10] to obtain information on the asymptotic behaviour of the solution to $(2.9)(2.10)$. The interesting thing here is the physical meaning of $H$ : recalling that $x$ is the volume variable, the first integral represents the total area of clusters. If the quadratic term were not present in the expression of $H$ this would exactly mean that the system tries to minimize its total area under the constraint (2.10) of constant total volume; the term $c^{2}$ should be thought of as a penalization of the monomer-monomer interaction. Another indication of this fact is given in [14], where it is shown that in order to go from the Becker-Döring system to the LifshitzSlyozov system by changing scale, it is necessary to turn off the reaction

$$
(1)+(1) \rightarrow(2)
$$

in the continuous limit, in order to avoid a singularity at small sizes (see [14] for details).

6.4. Intermediate asymptotics and self-similar behaviour. As was explained above, the Laplace transform provides explicit solutions when the initial data have an easily computable Laplace Transform, and the kinetic coefficients are homogeneous of degree $\alpha=0,1$, or 2 (so that the Laplace Transform at time $t$ satisfies a Partial Differential Equation of order $\alpha$, which can be explicitly integrated). This raises the question of the stability of these solutions with respect to both the initial data and the coefficients. If either the initial data or coefficients are slightly perturbed, it is conjectured in the applied literature that the relevant self-similar profile will continue to attract the solution for large time. Note that in the framework of parabolic equations, such a stability result was proved by renormalising arguments [7]. We are not aware however, of any rigorous proof of such a stability result for agglomeration systems. Very recently, some results on the stability with respect to initial data were obtained in [11], [2] and [27] for the cases $\alpha=0,1,2$. To give the reader an idea of the results, let us give the precise statement in [27] for the case of constant coefficients: 
Theorem 6.2. (Menon, Pego): Consider the solution $\mu_{t}$ to equation (4.5), with constant coefficient $k=2$. Define the size-biased distribution $F$ by

$$
F(x, t):=\frac{\mu_{t}([0, x[)}{\mu_{t}([0, \infty[)} .
$$

Assume there exists a number $\rho \in[0,1]$ and a function $L$ slowly varying at $\infty$ such that the initial mass of clusters smaller than $x$ is equivalent to $x^{1-\rho} L(x)$ as $x$ approaches infinity. Then there exists a rescaling factor $\lambda(t)$ which strictly increases to infinity, such that for any $x$ :

$$
F(t, \lambda(t) x) \rightarrow F_{\rho}(x) \quad \text { as } \quad t \rightarrow \infty,
$$

where $F_{\rho}$ is the Mittag-Leffler distribution (see [27] for the definition).

Similar results are available for the additive and multiplicative case.

This is in our opinion a major breakthrough in the rigorous study of intermediate asymptotics for agglomeration; it is quite likely that the method of proof developed in [27] (which of course uses the Laplace Transform in an essential way) will prove fruitful in more general contexts.

\section{Conclusion}

The mathematical theory of these systems has been burgeoning over the past few years, due to increasing recognition of the fact that:

- Obviously from the similarities between the involved interactions, many of the mathematical tools that had been previously developed for classical (mechanical) kinetic theory could be used to good advantage in this context.

- The underlying stochasticity of the process involved when chemical species react makes it very natural to view these evolutions as Markov processes.

- The striking analogy between the conjectured self-similar behaviour in coagulating systems and the self similarity property of some heavy-tailed stochastic processes cannot be fortuitous (in this connection, a decisive contribution was made recently in $[27])$.

We have strived here to convey the main mathematical ideas that are relevant to the field rather than expose state-of-the-art technical results. This paper being introductory, it is incomplete by nature. In particular, a vast area of activity which has not been touched upon here are the stochastic constructions which lead to these (deterministic) models. The reason for this is twofold: first, it falls out of the domain of competence of the author, and second, it is hard to improve upon the excellent introduction in [1].

It is of course very easy to complicate the models presented above by including other types of interaction, or new variables, or coupling them to some fluid-mechanical model, or other, and it is quite likely that for a large number of these "new" models, the classical techniques presented above would apply. If this could in some instances (very few in the author's opinion) be a worthwile exercise, one has to be very cautious about the physical relevance of the outcome.

It is our belief that most of the interesting developments in this area will come from an interplay between ideas from the theory of partial differential equations and the theory of stochastic processes, and our hope that this paper may have served to trigger the reader's interest. 


\section{REFERENCES}

[1] D. Aldous, Deterministic and stochastic models for coalescence (aggregation, coagulation): a review of the mean-field theory for probabilists, Bernoulli, 5:3-48, 1999.

[2] J. Bertoin, Eternal solutions to Smoluchowski's coagulation equation with additive kernel and their probabilistic interpretations, Ann. Appl. Probab., 12(2):547-564, 2002.

[3] J.M. Ball, On the asymptotic behavior of generalized processes, with applications to nonlinear evolution equations, J. Differential Equations, 27(2):224-265, 1978.

[4] E.A. Barbashin, On the theory of generalized dynamical systems, Moskov. Gos. Ped. Inst. Ucen. Zap., 2:110-133, 1948.

[5] J.M. Ball, J. Carr, and O. Penrose, The Becker-Döring cluster equations: Basic properties and asymptotic behaviour of solutions, Comm. Math. Phys., 104:657-692, 1986.

[6] R. Becker and W. Döring, Kinetische behandlung der keimbildung in ubersattigten damfern, Ann. Phys., (Leipzig) 24:719-752, 1935.

[7] J. Bricmont, A. Kupiainen, and G. Lin, Renormalization group and asymptotics of solutions of nonlinear parabolic equations, Comm. Pure Appl. Math., 47(6):893-922, 1994.

[8] S. Chandrasekhar, M. Kac, and R. Smoluchowski, Marian Smoluchowski: his life and scientific work, Polish Scientific Publishers, Warsaw, 2000.

[9] J. Carr and O. Penrose, Asymptotic behaviour of solutions to a simplified Lifshitz-Slyozov equation, Phys., D 124(1-3):166-176, 1998.

[10] J.F. Collet, T. Goudon, and A. Vasseur, Decay of monomer concentration in the Lifshitz-Slyozov equation, J. Stat. Phys., 108(1):341-359, 2002.

[11] M. Deaconu and E. Tanré, Smoluchowski's coagulation equation: probabilistic interpretation of solutions for constant, additive and multiplicative kernels, Ann. Sc. Norm. Pisa Cl. Sci., XXIX(4):549-579, 2000.

[12] S. Hariz and J.F. Collet, A modified version of the Lifshitz-Slyozov model, Letters in Applied Mathematics, 12:81-85, 1999.

[13] J.F. Collet, Quelques exemples de transitions de phase du premier ordre: les modèles d'aglomération, Matapli, 64:29-39, 2001.

[14] J.F. Collet, T. Goudon, F. Poupaud, and A. Vasseur, The Becker-Döring model and its LifshitzSlyozov asymptotics, SIAM J. Appl. Math., 62(5):1488-1500, 2002.

[15] J.F. Collet, T. Goudon, S. Hariz, F. Poupaud, and A. Vasseur, Some mathematical problems in cluster growth, to appear in IMA Volumes.

[16] M. Elimelech, J. Gregory, X. Jia, and R. Williams, Particle Deposition and Aggregation: Measurement, Modelling and Simulation, Colloid and Surface Engineering Series, Butterworth Heinemann, Oxford, 1995.

[17] S.K. Friedlander, Smoke, Dust and Haze, Wiley, 1977.

[18] Y. Gabellini and J.L. Meunier, Gel, self-similarity and universality in the discrete Smoluchowski equation with finite mass, J. Phys. A, Math. Gen., 25(13):3683-3700, 1992.

[19] J.D. Gunton, M.San Miguel, S.P. Sahni, The dynamics of first order phase transitions, in "Phase Transitions and Critical Phenomena", Domb, Lebowitz eds., 8:267-466,

[20] D. Henry, Geometric Theory of Semilinear Parabolic Equations, Lecture Notes in Mathematics, 840. Springer-Verlag, Berlin-New York, 1981.

[21] G.M. Hidy, Aerosols, Academic Press, 1984.

[22] PE. Jabin and B. Niethammer, On the rate of convergence to equilibrium in the Becker-Döring equations, J. Diff. Eq., 191(2):518-543, 2003.

[23] G.M. Hidy and J.R. Brock, Topics in Current Aerosol Research, Vols I,II,III, Pergamon Press, 1970.

[24] S.A. Kukushkin and A.V. Osipov, Kinetics of first-order phase transitions in the asymptotic stage, J. Exp. Theor. Phys., 86(6):1201-1208, 1998.

[25] L.D. Landau and E.M. Lifshitz, Statistical Physics, Course of Theoretical Physics, 5, Pergamon Press.

[26] I.M. Lifshitz and V.V. Slyozov, The kinetics of precipitation from supersaturated solid solutions, J. of Phys. and Chem. of Solids, 19:35-50, 1961.

[27] G. Menon and R.L. Pego, Approach to self-similarity in Smoluchowski's coagulation equations, preprint June 2003, arXiv:nlin.AO/0306047.

[28] B. Niethammer, R.L. Pego, The LSW model for domain coarsening: asymptotic behavior for conserved total mass, J. Statist. Phys., 104(5-6):1113-1144, 2001.

[29] G. Nicolis and I. Prigogine, Self-Organization in Nonequilibrium systems, Wiley, 1977.

[30] M.V. Smoluchowski, Drei vorträge über diffusion, brownsche bewegung und koagulation von kolloidteilchen, Phys. Z., 17:557-585, 1916. 
[31] M.V. Smoluchowski, Versus einer mathematischen theorie der kollö̈den lösungen, Z. Phys. Chem., 92:129-168, 1917.

[32] G. Toscani, Entropy production and the rate of convergence to equilibrium for the Fokker-Planck equation, Quart. Appl. Math., 57(3):521-541, 1999.

[33] V.V, Slyozov and V.V. Sagalovich, Diffusive decomposition of solid solutions, Sov. Phys. Usp., 30(1):23-45, 1997.

[34] P.G.J. Van Dongen and M.H. Ernst, Scaling solutions of Smoluchowski's coagulation equation, J. Statist. Phys., 50(1-2), 1988.

[35] J.J.L. Velàzquez, The Becker-Döring equations and the Lifshitz-Slyozov theory of coarsening, J. Statist. Phys., 92(1-2):195-236, 1998.

[36] C. Villani, Topics in optimal transportation, Graduate Studies in Mathematics, 58. American Mathematical Society.

[37] C. Wagner, Theorie der alterung von niederschlägen durch umlösen, Z. Elektrochem. 65:581$594,1961$. 